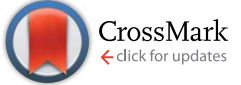

Cite this: J. Mater. Chem. A, 2015, 3 , 3399

Received 7th December 2014 Accepted 16th December 2014 DOI: $10.1039 / c 4 t a 06719 c$

www.rsc.org/MaterialsA

\section{The effect of oxygen induced degradation on charge carrier dynamics in P3HT:PCBM and Si- PCPDTBT:PCBM thin films and solar cells $\uparrow$}

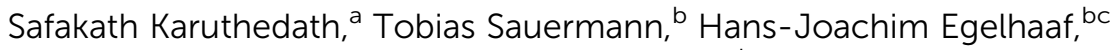 \\ Reinhold Wannemacher, ${ }^{a}$ Christoph J. Brabec ${ }^{d}$ and Larry Lüer ${ }^{\star a}$
}

\begin{abstract}
Due to their light weight, transparency and flexibility, organic photovoltaic (OPV) devices are ideal for building integration. As this application requires solar cell life times of more than twenty years and oxygen ingress cannot be avoided at competitive cost on this time scale, OPV modules must be intrinsically stabilized against photo-oxidation. To this end, the mechanism of rapid performance loss of OSCs due to oxygen-induced degradation must be understood. Here, we combine transient absorption experiments with electrical studies in P3HT:PCBM and Si-PCPDTBT:PCBM thin films and solar cells after controlled photo-oxidation, studying charge carrier dynamics on the femtosecond to millisecond time scale. We find that oxygen-induced degradation does not significantly influence charge generation, while its influence on charge recombination is strong in both materials. A dramatic retardation of charge recombination already at low levels of oxygen-induced degradation is attributed to a substantial reduction of charge mobilities. We also observe a significant increase of the background concentration of charge carriers with the level of degradation, which leads to a crossover from second order towards pseudo-first order recombination behaviour. Extraction is shown to be retarded even more strongly than recombination, possibly by a reduction of the extraction field by the background carriers. Overall, the recombination yield is increased with degradation, explaining the strong performance loss already at low degradation levels.
\end{abstract}

\section{Introduction}

Polymer based organic solar cells (OSCs) can be mass-produced by solution-based roll-to-roll printing, which consumes far less energy than needed for the production of silicon-based solar cells. $^{1-7}$ Further advantages of OSCs are transparency, flexibility and colour tunability, making them apt for new markets like consumer products and architectural integration. ${ }^{8}$ For the latter, lifetimes exceeding some 20 years are mandatory. However, the stability of OSCs is compromised by several degradation mechanisms, which occur at different rates, according to packaging and environmental conditions. Humidity is a well-known factor for device degradation by electrode oxidation; it has been shown that the introduction of the inverted architecture led to improvements. ${ }^{9}$ Another

${ }^{a}$ IMDEA Nanociencia, C/Faraday, 9, 28049 Cantoblanco, Madrid, Spain. E-mail: larry. luer@imdea.org

${ }^{b}$ Belectric OPV GmbH, Landgrabenstr. 94, 90443 Nürnberg, Germany

${ }^{c}$ Bavarian Center for Applied Energy Research, Fürther Str. 250, 90429 Nürnberg, Germany

${ }^{d}$-MEET, Friedrich-Alexander University, Martensstr. 5, 91058 Erlangen, Germany

$\dagger$ Electronic supplementary information (ESI) available. See DOI: $10.1039 / \mathrm{c} 4 \mathrm{ta} 06719 \mathrm{c}$ mechanism affecting mainly the active layer is photo-oxidation. $^{\mathbf{1 0 , 1 1}}$ Oxygen exclusion by means of high quality encapsulation, which is standard in small area AMOLED displays for example, is not viable for OSCs where the cost of packaging must not exceed $5 € \mathrm{~m}^{-2}$. Therefore, research efforts have to be directed towards intrinsically stabilizing the OSC active layer against photo-oxidation. In poly(3-hexylthiophene) (P3HT) films, it has been shown that irradiation in oxygen atmosphere causes irreversible bleaching of the ground state absorption, attributed to oxygen induced chain scission and formation of stable photoproducts, probably in a radical chain reaction. ${ }^{12}$ In OSCs based on P3HT and [6,6]-phenyl- $\mathrm{C}_{61}$-butyric acid methyl ester (PCBM), oxygen-induced degradation causing $2 \%$ ground state absorption loss leads to a $70 \%$ reduction in the short circuit current. ${ }^{13}$ Therefore, the observed strong effect on $J_{\mathrm{SC}}$ cannot be attributed to a mere loss of initially excited states due to absorption loss. Considering photoelectric conversion as a sequence of the elementary steps: (i) absorption, (ii) exciton diffusion to the bulk heterojunction, (iii) charge formation by exciton dissociation, and (iv) charge extraction, oxygen-induced degradation is therefore expected to cause a strong reduction in at least one of the latter three processes.

With respect to the second step, it has been shown recently that at elevated levels of degradation (5-20\% of optical 
absorption loss), an ultrafast exciton quenching channel is active that might explain at least partially the reduction of the overall photovoltaic yield. ${ }^{\mathbf{1 4}}$ However, the extent of exciton quenching is by far not strong enough to account for the drastic loss of short circuit current; moreover, it is not clear whether this process plays any role at lower levels of degradation.

In order to shed more light on the effect of photo-oxidation on the two elementary steps involving charge carriers, we present here a transient absorption (TA) study of photo-induced charges in thin films and OSCs, comprising two prominent active materials, namely the blends of poly-3-hexylthiophene with [6,6]-phenyl- $\mathrm{C}_{61}$-butyric acid methyl ester (P3HT:PCBM) and of the low-band gap copolymer poly[(4,4 0-bis(2-ethylhexyl) dithieno[3,2-b:2 $\quad 0,3 \quad 0$-d $]$ silole)-2,6-diyl-alt-(4,7-bis(2-thienyl)2,1,3-benzothiadiazole)-5,5 0-diyl] (Si-PCPDTBT) with PCBM. The TA bands of photo-induced charges in P3HT and SiPCPDTBT are well characterized. ${ }^{\mathbf{1 5 , 1 6}}$

An overview of the multitude of possible effects of photodegradation on the charge carrier dynamics in bulk heterojunction (BHJ) films is conveniently obtained on the basis of a simplified kinetic model for charge carrier density $n$ in the active layer of a device of thickness $d$, applying idealized assumptions such as homogeneous medium, second order recombination, and constant built-in field $E_{\mathrm{bi}}$ :

$$
\frac{\mathrm{d} n}{\mathrm{~d} t}=G-k_{\mathrm{L}} n n-\mu n \frac{E_{\mathrm{bi}}}{d},
$$

where $\mu$ is the effective charge carrier mobility, $G$ is the photoinduced generation of extractable charge carriers, and $k_{\mathrm{L}}$ is the bimolecular charge recombination coefficient, assuming Langevin recombination

$$
k_{\mathrm{L}}=2 \mathrm{e} \mu_{\mathrm{eff}} f_{\mathrm{LV}} /\left(\varepsilon \varepsilon_{0}\right)
$$

Herein, $\mu_{\text {eff }}=\mu_{\mathrm{e}}+\mu_{\mathrm{h}}$ is the sum of the mobilities for positive and negative charges, respectively, $\varepsilon$ is the relative dielectric constant, $\varepsilon_{0}$ is the dielectric constant in a vacuum, and $f_{\mathrm{LV}}$ accounts for sub-Langevin recombination, as often observed in BHJ active layers. ${ }^{17}$

The short circuit current $J_{\mathrm{SC}}$ is obtained at a steady state, i.e., $\mathrm{d} n / \mathrm{d} t=0$.

$$
J_{\mathrm{SC}}=\mu n_{\mathrm{SS}} E_{\mathrm{bi}} / d,
$$

where the steady state carrier concentration $n_{\mathrm{SS}}$ is obtained by solving eqn (1a) for $\mathrm{d} n / \mathrm{d} t=0$ :

$$
n_{\mathrm{SS}}=\left[-\mu \frac{E_{\mathrm{bi}}}{d}+\sqrt{\left[\mu \frac{E_{\mathrm{bi}}}{d}\right]^{2}+4 k_{\mathrm{L}} G}\right] /\left(2 k_{\mathrm{L}}\right)
$$

Following eqn (3), the OSC performance loss can be caused by a reduction of charge carrier generation rate $G$, charge carrier mobility $\mu$, and built-in field $E_{\mathrm{bi}}$ or by an increase of the nonLangevin factor $f_{\mathrm{LV}}$. The charge carrier mobility appears in both recombination and extraction terms, but in the case of bimolecular recombination its effect on extraction dominates.
A reduction of $G$ can be caused either by bleaching of absorption, enhanced exciton deactivation, e.g. by quenching by oxidation products, or by an increase of geminate recombination. Non-geminate recombination has been shown to be negligible in annealed films of P3HT:PCBM, but might be enhanced by degradation-induced changes in the conductive pathways towards the extracting electrodes, e.g., by broadening the distribution of the density of transport states. ${ }^{18}$ Formally, this corresponds to a decrease in carrier drift velocity from the bulk towards the electrodes. A decrease of charge carrier mobility would also lead to reduced recombination, as actually observed by transient absorption measurements on degraded MEH-PPV:PCBM devices and by TPV measurements on degraded P3HT:PCBM devices, both cells being of regular architecture. ${ }^{19,20}$ However, in the case of bimolecular recombination, the overall effect of reduced mobility on short circuit current would be detrimental by virtue of an increase of $n_{\mathrm{SS}}$. The charge extraction yield can also be reduced by an increased charge recombination rate $k_{\text {rec }}$ ("trap-induced recombination"); in the framework of eqn (3), trap-induced recombination would either be caused by reduced mobility $\mu$ or by an increase of the non-Langevin factor $f_{\mathrm{LV}}{ }^{21}$ Finally, a reduction of the built-in field $E_{\mathrm{bi}}$ in a large part of the bulk heterojunction due to the defect-induced creation of a space charge layer has been proposed as the reason for the reversible performance loss in P3HT:PCBM solar cells upon doping by oxygen and may also be the reason for the reduced charge carrier extraction rate in irreversibly oxidized samples. ${ }^{13}$

The distinction between the above mentioned reasons for short circuit current loss is best done in the time domain, because the corresponding loss mechanisms lead to clearly different charge carrier decay kinetics. Furthermore, transient absorption (TA) is preferred over electrical transients, because different from electrical methods relying on charge motion, TA is sensitive also to immobile charges, thus being able to detect the degradation-induced immobilization of part of the carriers.

\section{Experimental section}

The characteristics of the materials for the photoactive layer were as follows: PCBM, purity 99.5\%; P3HT, $M_{\mathrm{w}}=65.5 \mathrm{kD}$, PDI $=2.04$; Si-PCPDTBT, $M_{\mathrm{w}}=52.8 \mathrm{kD}$, PDI $=2.85$. Solutions of P3HT : PCBM $(1: 0.8)$ and Si-PCPDTBT : PCBM $(1: 2)$ were prepared in water-free $o$-xylene $(97 \%)$ by stirring at $80{ }^{\circ} \mathrm{C}$ and $120^{\circ} \mathrm{C}$, respectively, overnight. These solutions were used for both solar cell devices in an inverted stack and neat films on glass substrates. The solar cells were made by applying a holeblocking layer on an ITO-glass substrate, followed by the photoactive layer and the electron-blocking layer (EBL) with a transparent electrode. For the EBL, the commercially available HTL Solar 246 from Heraeus was used and the top electrode was realized by using the commercially available high conductive PEDOT PHCV6 (in isopropanol, 99.5\% purity), combined with an evaporated silver grid electrode on top. This architecture ensures that the degradation of the active layer in the solar cell is the same as the degradation of the neat film, since the electrodes are gas-diffusion-open. ${ }^{13}$ The architecture also ensures 
that transient absorption spectroscopy is sensitive across the device thickness, because TA can be measured in transmission, avoiding destructive interference of the probe light close to the reflecting electrodes. ${ }^{22}$ All organic layers were applied by doctor blading. Care was taken that doctor blading of the polymer:fullerene layers occurred under the same conditions for solar cells and neat films, which led to a thickness of $250 \mathrm{~nm}$ for P3HT:PCBM layers and $150 \mathrm{~nm}$ for Si-PCPDTBT:PCBM layers.

Prior to degradation, both types of devices were annealed at $140{ }^{\circ} \mathrm{C}$ (P3HT) and $130{ }^{\circ} \mathrm{C}$ (Si-PCPDTBT) for 5 minutes in a glovebox to achieve reproducible morphologies of the films and active layers of the solar cells. Afterwards all devices were exposed to AM1.5 irradiation in dry synthetic air $\left(80 \% \mathrm{~N}_{2}, 20 \%\right.$ $\mathrm{O}_{2}, 0 \% \mathrm{H}_{2} \mathrm{O}$ ) for different time periods until the desired degradation was achieved. Subsequently, the degraded devices were returned into the glovebox and annealed at $130{ }^{\circ} \mathrm{C}$ for 5 minutes to remove the residual oxygen doping. The progress of degradation was monitored by UV/vis-absorption spectroscopy and the loss of optical density was then correlated with the loss in $J_{\mathrm{SC}}$ of the solar cells.

Absorption spectra of the pristine and degraded samples are supplied in the ESI. $\dagger$ Both films, P3HT:PCBM and SiPCPDTBT:PCBM, were again annealed under vacuum at $140{ }^{\circ} \mathrm{C}$ and $130{ }^{\circ} \mathrm{C}$, respectively, for 5 minutes in a cryostat (Oxford OPTISTAT), equipped with a heater, before each experiment, in order to make sure that the reversible oxygen doping is removed and only irreversible degradation effects are measured.

Microsecond transient absorption measurements were performed by exciting the annealed samples at room temperature under high vacuum with a $532 \mathrm{~nm}$ (7 mm spot size) continuous wave Nd:YAG laser modulated using a chopper (at $1: 1$ duty cycle) on which the beam was sharply focused to realize illumination rise and fall times of around $750 \mathrm{~ns}$. On the sample, the pump laser had a spot size of $7 \mathrm{~mm}$ with a constant intensity (to within 15\%) throughout the spot. A $970 \mathrm{~nm}$ (2 mm spot size) $120 \mathrm{~mW}$ LED was used for probing the photo-excitation dynamics of polarons in both P3HT:PCBM and SiPCPDTBT:PCBM. The transient absorption signal was detected using an amplified Si-photodiode (Thorlabs PDA 10A EC) equipped with a $850 \mathrm{~nm}$ long pass filter in order to reject scattered pump light and fluorescence. The electrical signal from the photodiode was then sampled with an oscilloscope (Picoscope 6424) connected to a PC using custom built Python software. Data evaluation was done using custom built Python software.

The absorption maxima of positive polarons in P3HT and SiPCPDTBT are at $980 \mathrm{~nm}$ and $1300 \mathrm{~nm}$, respectively. ${ }^{\mathbf{1 5 , 1 6}}$ At our probing wavelength of $970 \mathrm{~nm}$, both materials show sufficiently large polaron absorption cross-sections with negligible superposition of other long-lived photo-excited states. On a microsecond time scale, the contribution of singlet exciton absorption, which is known to overlap strongly with polaron absorption at $970 \mathrm{~nm}$, can be safely neglected because excitons, even if they do not reach an interface, are quenched intrinsically by radiative and non-radiative processes on a few nanosecond time scale. ${ }^{15}$ This notion holds also for delayed exciton regeneration due to charge recombination: due to the short lifetime of excitons, the stationary exciton concentration caused by microsecond charge recombination will be negligible. Therefore, we can consider the $\Delta A(970 \mathrm{~nm})$ time traces, that we will discuss in the remainder of the manuscript, as proportional to the concentration of polarons (positive or negative) in the P3HT or Si-PCPDTBT phases, respectively.

Femtosecond transient absorption (TA) spectra have been obtained with a regeneratively amplified system at $1 \mathrm{kHz}$ repetition rate (Clark-MXR CPA2101). The $775 \mathrm{~nm}$ pulses were split into two parts: one part was frequency doubled using an LBO crystal (pump pulses) and the other part was focused onto a 2 $\mathrm{mm}$ sapphire plate, producing a femtosecond white light super continuum from 420-1600 $\mathrm{nm}$ (probe pulses). The probe pulses were focused onto the sample (about $150 \mu \mathrm{m}$ diameter), and overlapped with the pump pulse (about $300 \mu \mathrm{m}$ diameter), the delay time of which was controlled via a mechanical translation stage and a corner cube. After transmission through the sample, the probe pulses were sent to a prism spectrometer (Entwicklungsbüro Stresing $\mathrm{GmbH}$ ) with a CCD array (256 pixels, VIS-enhanced InGaAs, Hamamatsu Photonics Inc.). Data acquisition and modelling were done by custom built Python software, using open source packages Matplotlib, PyQt4, SciPy, Pyserial, among others.

\section{Results}

In Fig. 1a and c, we show the $J-V$ characteristics of our solar cells after specified times of irradiation using a sun simulator under dry synthetic air. To relate the electrical performance to the optical absorption of the devices, we compare in Fig. $1 \mathrm{~b}$ and $\mathrm{d}$ the short circuit current $\left(J_{\mathrm{SC}}\right)$ to the normalized optical density (OD). After 240 minutes of irradiation, the $J_{\mathrm{SC}}$ is irreversibly reduced to $35 \%$ (25\%) of the original value, while the loss in OD is only around $2 \%(3.5 \%)$ for P3HT:PCBM and SiPCPDTBT:PCBM, respectively. The vast majority of the electrical performance loss of the solar cells can therefore not be ascribed to a loss in optical absorption, in agreement with earlier findings. ${ }^{12,13}$ Oxygen-induced photo-degradation obviously affects mainly the internal quantum efficiency (IQE). As discussed in the Introduction, we apply transient absorption spectroscopy in films and OSCs to single out the factors that contribute to the loss of IQE. We will first analyse picosecond transient spectra to evaluate the effect of degradation on charge carrier formation and then turn to microsecond transients to elucidate the effect of degradation on charge carrier recombination and extraction.

In OPV devices, charge carrier generation involves exciton diffusion and dissociation at the $\mathrm{D} / \mathrm{A}$ interface, a process known to occur on a femtosecond to picosecond time scale. ${ }^{\mathbf{1 5}}$ Depending on material and morphology, charge carrier separation, occurring on a picosecond time scale, can be opposed by geminate recombination. ${ }^{18}$ In order to study whether photooxidation has an influence on exciton diffusion and/or geminate recombination, we performed femtosecond transient absorption (TA) spectroscopy. In Fig. 2a, we show a TA spectrum of a pristine Si-PCPDTBT:PCBM film, taken at low enough pump intensity so that only processes are observed that also occur under CW solar irradiation. In agreement with previous 

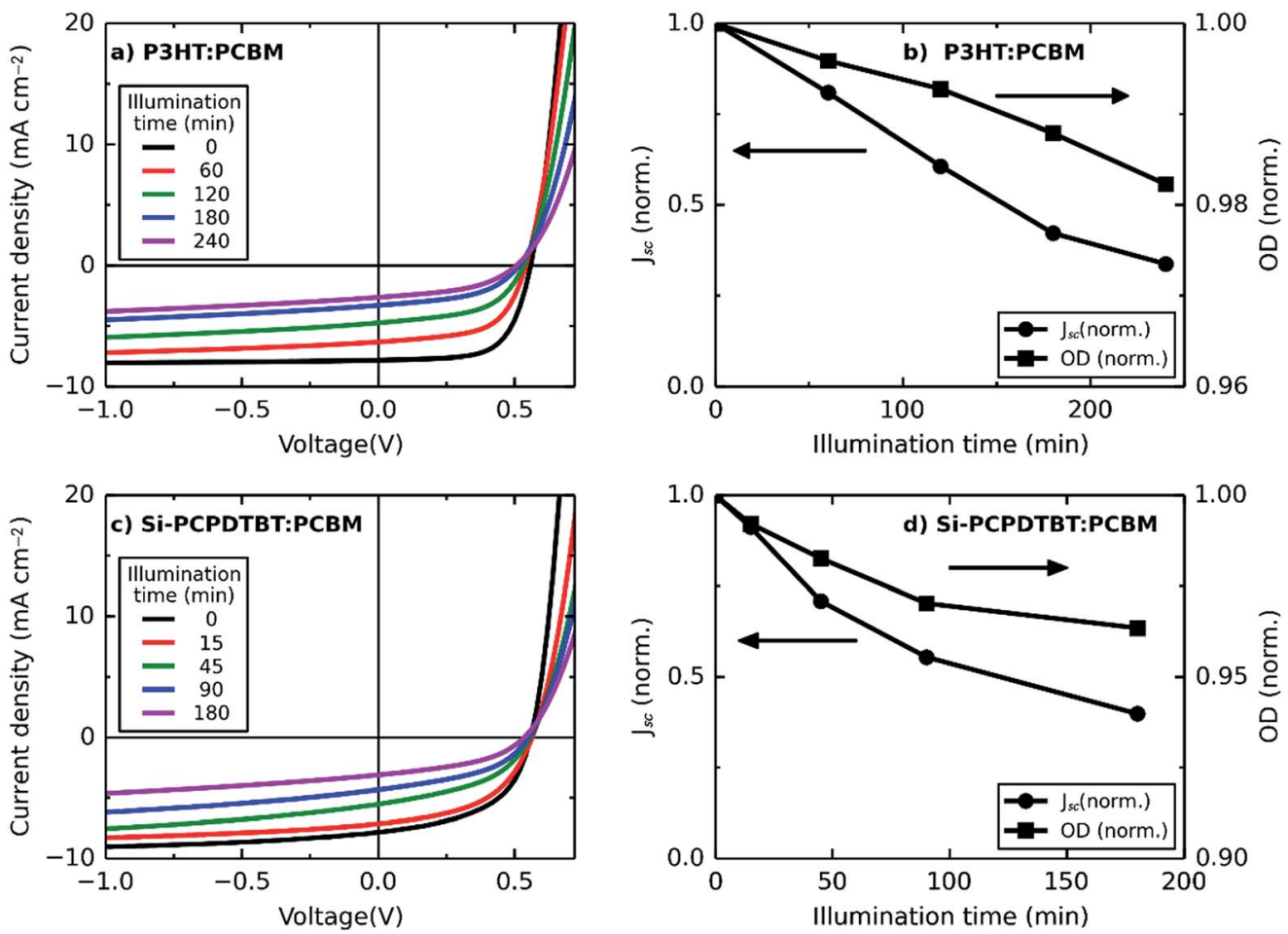

Fig. 1 Current density-voltage ( $\mathrm{J}-\boldsymbol{V}$ ) characteristics (1st column) and a comparison of optical density (OD) losses and electrical losses (second column) for P3HT:PCBM (panels a and b) and Si-PCPDTBT:PCBM (panels $\mathrm{c}$ and d), during degradation of the devices by irradiation with the light of a sun simulator under synthetic air.

reports, we find a photo-induced absorption (PA) band of polarons at $1.0 \mathrm{eV}$, a very short lived band at $0.85 \mathrm{eV}$, assigned to singlet excitons, and a transient photobleaching (PB) band around $1.7 \mathrm{eV} .^{16}$ The time-resolved TA spectrum can be used to obtain the absolute charge carrier yield as a function of pumpprobe delay time. To this end, we reproduce the TA spectrum by a superposition of 3 spectra, each with an associated lifetime following a first order sequential reaction of type $0 \rightarrow \mathrm{A} \rightarrow \mathrm{B} \rightarrow$ $\mathrm{C} \rightarrow 0$. Please note that this procedure is purely mathematical and does not involve any photophysical assumptions. Therefore, the spectra of A, B, and C do not correspond to real photophysical states (singlet excitons and charged states) but to superpositions of these, and therefore are called evolutionassociated differential spectra (EADS 0,1 , and 2, respectively, thin black curves in Fig. 2b). The contribution of singlets and charged states to each EADS can be obtained by spectral modelling. Only at this point, photophysical knowledge enters the procedure, essentially by requiring (i) that different states have different PA bands, (ii) that there are only 2 distinct photoexcited states in our spectral window, namely singlets and charged states of the donor polymer, and finally (iii) that both singlets and charged states share the same transient photobleach. The procedure results in individual spectra for singlets and charged states (dashed and dotted curves in Fig. 2b, respectively), the spectral weights of which corresponding to their concentrations (for details, see ESI†).

It is found that EADS 0, representing the excitation state right after the pump pulse, is composed of both singlet and charged states, whereas the subsequent EADS contain nearly exclusively charged states. Hence, we can associate the transition from EADS 0 to 1 with singlet exciton dissociation $(1 \mathrm{ps}$ lifetime) and the transition from EADS 1 to 2 with geminate recombination (92 ps lifetime). In Fig. 2c, the PB region of EADS 2 is shown at different degradation levels, representative of the amount of charge carriers that survive geminate recombination. No significant dependence of the spectral weight on degradation can be found. It is important to note that the presence of $\mathrm{PB}$ in Fig. 2 is insensitive to the type of excitation: a conjugated segment of Si-PCPDTBT which is excited cannot contribute to ground state absorption, irrespective of whether the excitation is neutral or charged. Since both singlets and polarons share the same $\mathrm{PB}$, it is possible to quantitatively obtain the time-resolved exciton and polaron yields. The polaron yield after geminate recombination (extracted from EADS 2) is given in the inset of Fig. 2c for all degradation levels, not showing any significant degradation influence. Hence, at degradation levels relevant for device operation, neither exciton diffusion nor geminate recombination are significantly influenced by photo-oxidation. This finding, obtained by femtosecond spectroscopy, is confirmed by charge dynamics during illumination with very weak microsecond pulses at 0.1 absorbed sun, see Fig. 2 d. It is found that the rise of polaron absorption during the first microseconds of irradiation is independent of degradation. According to eqn (1), we can neglect the recombination term at early times while the charge concentration is still low, so the initial rise of charge density is a measure of the generation of 

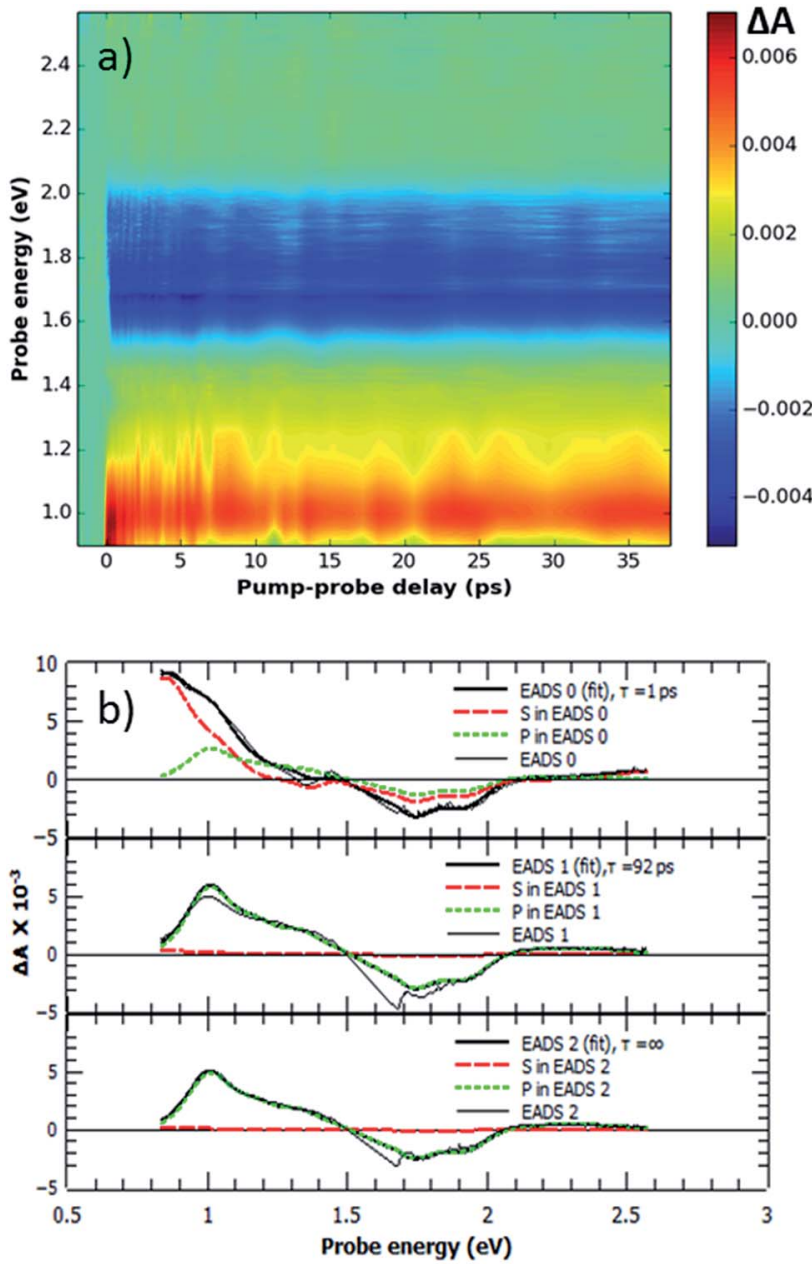
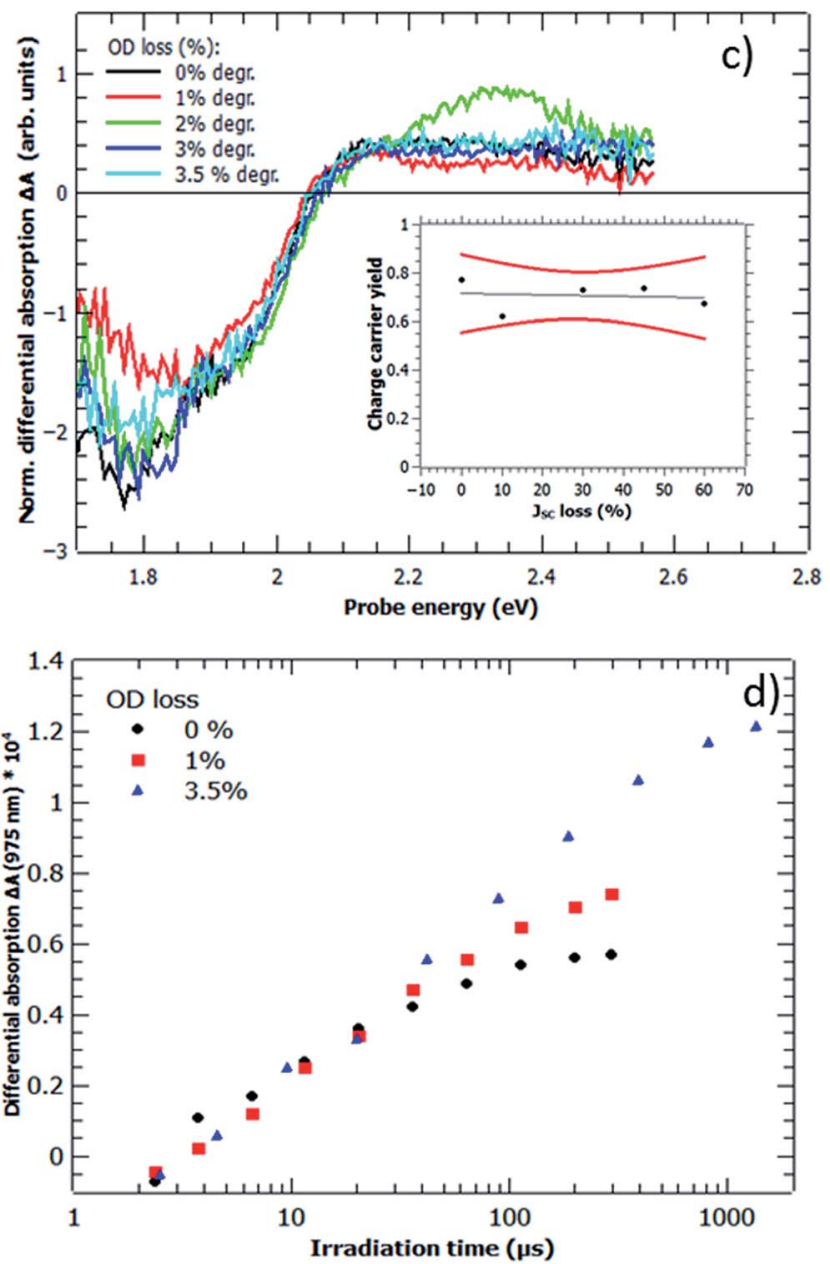

Fig. 2 Charge carrier generation in Si-PCPDTBT:PCBM as a function of degradation. (a) Femtosecond transient absorption (TA) spectrum of the pristine sample upon pumping with $150 \mathrm{fs}$ pump pulses at $387 \mathrm{~nm}$, shown in a false color representation (see the color bar). (b) Evolution associated differential spectra (EADS), as obtained from a global analysis of (a) using a 3 sequential step model (thin solid curves, fitted lifetimes are given in the legend), spectral fits to the EADS (thick solid curves) and contributions to fits from singlet and polaron states (dashed and dotted curves, respectively). (c) Bleach part of long-lived EADS 2 at various degradation levels, normalized to the total spectral weight of EADS 0 at the same degradation level. The inset shows the total yield of polarons surviving geminate recombination. Each point (for the respective $J_{S C}$ loss) is obtained by dividing the spectral weight of the polaron contribution in EADS 2 by the total spectral weight of EADS 0 . (d) Charge density increase (measured at $975 \mathrm{~nm}$ ) upon switching on irradiation at 0.1 absorbed sun, at different degradation levels.

extractable carriers. An analogous study in P3HT:PCBM yields a similar result (see ESI $\dagger$ ).

In Fig. 3, decay traces of the polaron absorption band are shown on a micro- to millisecond time scale for the same films as in Fig. 2 upon light-off, after a long enough irradiation period to reach stationary charge carrier concentrations at the respective degradation levels (between 5 and $83 \mathrm{~ms}$ ). According to eqn (1), these decay curves allow to specifically obtain recombination information, since both extraction and generation are absent, due to the absence of electrodes and irradiation, respectively. We define $t=0$ as the time of termination of illumination. In order to visualize details of the decay at various times and signal levels in a single graph, we chose a double logarithmic representation of the data. In pristine P3HT:PCBM (Fig. 3a), a strong intensity dependence of the decay curves is found: while the starting concentrations at $t=1 \mu$ s cover nearly an order of magnitude, after $1 \mathrm{~ms}$ decay time all curves are within a factor of 2. This behaviour is indicative of a pure bimolecular reaction with equal concentrations of positive and negative carriers, as expected if only photo-induced carriers are present. When this condition is met, eqn (1) holds, and the time integral evaluates to an intensity independent, hyperbolic dependence of concentration on time for long times. This behaviour is experimentally confirmed in Fig. 3a, in agreement with previous reports on pristine P3HT:PCBM. ${ }^{22}$

Photo-degradation of the P3HT:PCBM films causes a drastic change in the charge recombination dynamics, compare Fig. 3a and b. First, note an overall reduction of the recombination rate: at $26 \mathrm{~mW} \mathrm{~cm}^{-2}$, it takes $200 \mu$ s for the $2 \%$ degraded sample to lose half of the initial carrier concentration while in the pristine case, as much as 5/6 of the initial carriers are lost at the same time. Second, the long-term behaviour is entirely different 

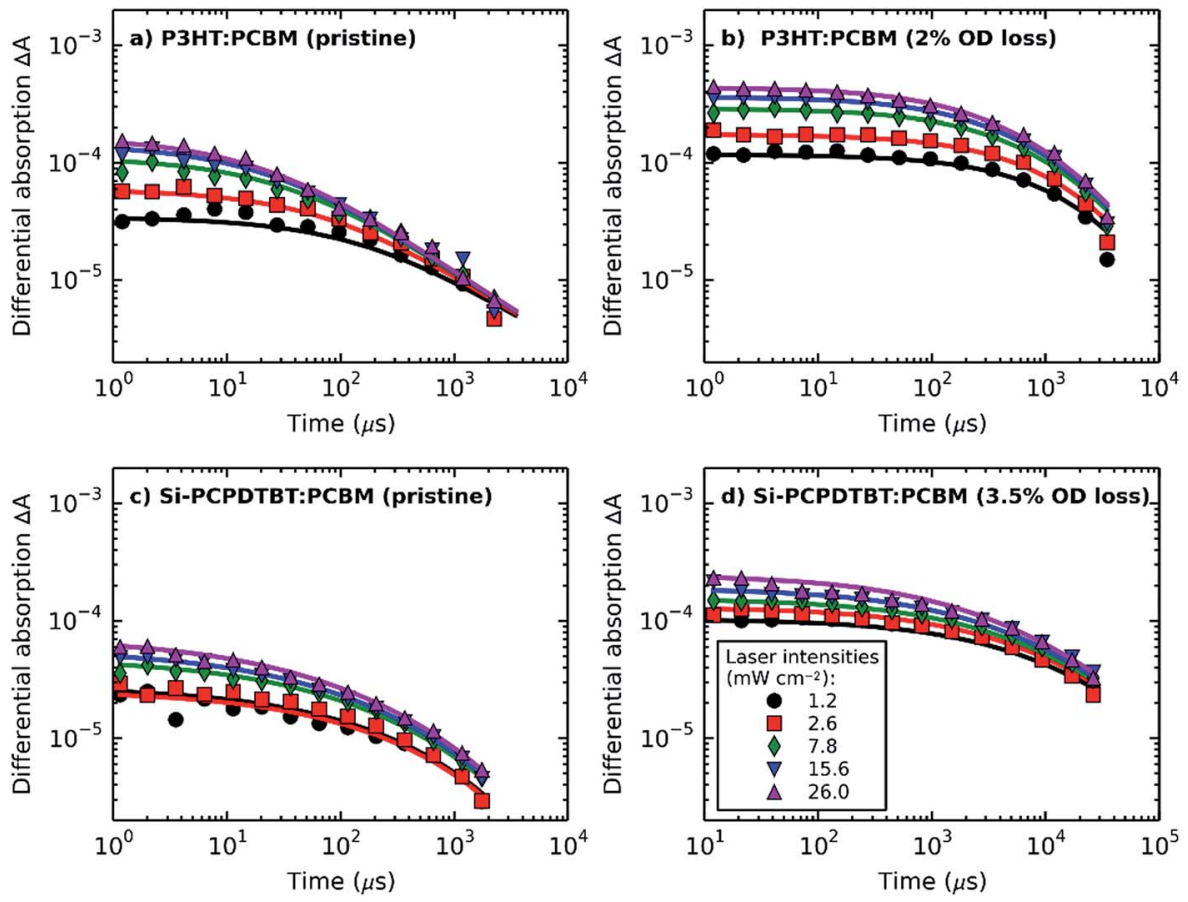

Fig. 3 Charge transients in polymer:PCBM films at different light intensities at $970 \mathrm{~nm}$ probing wavelength, upon termination of illumination. Panels (a) and (b) show transients of P3HT:PCBM at degradation levels of $0 \%$ and $2 \%$, respectively. Panels (c) and (d) are transients of SiPCPDTBT:PCBM at degradation levels of $0 \%$ and $3.5 \%$, respectively. For panels (a-c), the samples were illuminated for $5.5 \mathrm{~ms}$ before light-off, at a repetition rate of $91 \mathrm{~Hz}$; for panel (d), the illumination period was $83.3 \mathrm{~ms}$ at $6 \mathrm{~Hz}$ repetition rate. The intensity of the laser ( $\lambda=532 \mathrm{~nm})$ during light-on is given in the legend.

for degraded P3HT:PCBM: there is no asymptotic straight line as in the case of the pristine sample, the curves rather tend towards an infinite slope, which is typical for the double logarithmic representation of a (stretched) exponential function. Moreover, the curves in Fig. $3 \mathrm{~b}$ do not collapse into a unitary, intensity independent long-time behaviour as in Fig. 3a. Both observations are in agreement with a reduction of the reaction order towards pseudo-first order, which is usually caused by excess concentrations of either positive or negative carriers.

Irreversible degradation in Si-PCPDTBT:PCBM films leads to a similar behaviour as in P3HT:PCBM, namely, an overall reduction of the recombination rate and a transition towards a lower recombination order. However, note that even in the pristine case, Si-PCPDTBT:PCBM does not exhibit perfect second order behaviour, that is, the single decay curves at different intensities do not collapse into a unitary, intensityindependent straight line for long times.

In conclusion, the main effect of oxygen-induced degradation on both P3HT:PCBM and Si-PCPDTBT:PCBM films is a strong retardation of charge recombination and a concomitant transition from reaction orders close to two towards reaction orders close to unity. These results are in accordance with the results presented by Deibel et al. ${ }^{19}$ They are clearly in contradiction to any defect enhanced recombination rate as reason for the degradation of device performance. ${ }^{23}$

\section{Discussion}

In the following, we will describe the qualitative observations of the previous section by a quantitative kinetic model. Since the measurements in Fig. 2 and 3 have been performed in films without charge extraction layers and electrodes, recombination via device related shunt resistances and charge extraction need not be considered. The rate equation describing charge carrier dynamics thus becomes

$$
\frac{\mathrm{d} n}{\mathrm{~d} t}=G-k_{\mathrm{L}}(t)\left[n(t)+c_{\mathrm{b}}\right] n(t),
$$

Eqn (4) refines the simple model represented in eqn (1) in two aspects. First, we allow for a population of mobile background carriers of concentration $c_{\mathrm{b}}$, which arises from degradation and might result from degradation-induced trap states, most likely for electrons (for details and a complete derivation see ESI $\dagger$ ). Second, we consider the fact that charge carrier mobility in P3HT:PCBM depends on the occupied density of states and therefore, in time-resolved experiments, implicitly also on time. ${ }^{22,24}$ This causes the bimolecular recombination coefficient to be time-dependent, which is usually taken into account by defining

$$
k_{\mathrm{L}}(t)=k_{\mathrm{L}}^{0}(t / 1 \mu \mathrm{s})^{\gamma},
$$

where $k_{\mathrm{L}}^{0}$ is the value of the bimolecular recombination coefficient (in $\mathrm{cm}^{3} \mathrm{~s}^{-1}$ ) at $t=1 \mu \mathrm{s}$, and $\gamma$ is the dispersive 
parameter. ${ }^{22,25,26}$ Note that generally, care must be taken when extracting device relevant information from decay curves: once the carrier population has decayed far below the steady state value at 1 sun, $n_{\mathrm{Ss}}$ ( 1 sun), the mobilities and hence the observed recombination times will be different from those under 1 sun CW illumination. For this reason, we report and discuss the value for $k_{\mathrm{r}}^{0}$ at $t=1 \mu \mathrm{s}$, when the remaining carrier population is still close to $n_{\mathrm{Ss}}$. Inserting the Beer-Lambert law, $A$ $=\sigma c d$, into eqn (4) yields

$$
\frac{\mathrm{d} \Delta A}{\mathrm{~d} t}=\tilde{G}-\tilde{k}_{\mathrm{L}}(t)\left(\Delta A+A_{\mathrm{b}}\right) \Delta A,
$$

where $\tilde{G}=\sigma d G, \tilde{k}_{\mathrm{L}}(t)=k_{\mathrm{L}}(t) / \sigma d$ (see ESI $\dagger$ for a complete derivation). Herein, $\sigma \approx 1 \times 10^{-16} \mathrm{~cm}^{2}\left(\sigma \approx 3 \times 10^{-17} \mathrm{~cm}^{2}\right)$ are the absorption cross-sections of polarons in the P3HT (SiPCPDTBT) phase at $970 \mathrm{~nm}, d=250 \mathrm{~nm}$ is the film thickness, and $A_{\mathrm{b}}$ is the absorption of the background carriers of concentration $c_{\mathrm{b}}$. Eqn (6) can be directly used to fit the transient absorption traces in Fig. 2 and 3.

Fitting eqn (6) to the rising curves after switching on the illumination in Fig. 2, we obtain the degradation-dependent generation parameter $\tilde{G}$. Dividing this parameter by the incident photon flux gives us the polaron yield before non-geminate recombination (per absorbed photon). For P3HT:PCBM, we find polaron yields between 0.6 and 0.7 , not significantly depending on the degradation level (black symbols in Fig. 4a). An analogous analysis for Si-PCPDTBT (Fig. 2d) was not possible because of the time resolution limit of our microsecond TA spectrometer. However, picosecond TA data, available for both P3HT:PCBM and Si-PCPDTBT, confirm that there is no significant change of the polaron yield with degradation (see red and green symbols in Fig. 4a; for details see ESI $\dagger$ ).

Fitting the decay traces after termination of illumination (Fig. 3) we can set $G=0$ in eqn (6). As can be seen by inspecting the solid curves in Fig. 3 and the additional graphs shown in the ESI, $\dagger$ eqn (6) describes very well the charge recombination in both P3HT:PCBM and Si-PCPDTBT:PCBM, at all degradation levels including the pristine case, with one single parameter set valid for all irradiation intensities (i.e., all fits given in Fig. 3 are global fits). Fig. 4 displays the evolution of the recombination coefficient $k_{\mathrm{L}}^{0}$ (given at $t=1 \mu \mathrm{s}$ ), of the exponent $\gamma$, and of the background concentration $c_{\mathrm{b}}$ as a function of degradation, as obtained from the fits (panels b, c, and d, respectively). We find that photo-degradation strongly reduces $k_{\mathrm{L}}^{0}$ for both P3HT:PCBM and Si-PCPDTBT:PCBM. At a $J_{\mathrm{SC}}$ loss of around $60 \%, k_{\mathrm{L}}^{0}$ is reduced by more than an order of magnitude. According to Langevin's formula (see eqn (1b) and ESI $\dagger$ ), this suggests a drastic reduction of charge carrier mobility $\mu$ already at early stages of degradation. ${ }^{27}$

Fig. 4c displays the dispersiveness parameter $\gamma$. A value of $\gamma$ $=0$ would correspond to a time-independent mobility as implicitly assumed in eqn (1), leading to a slope of -1 as
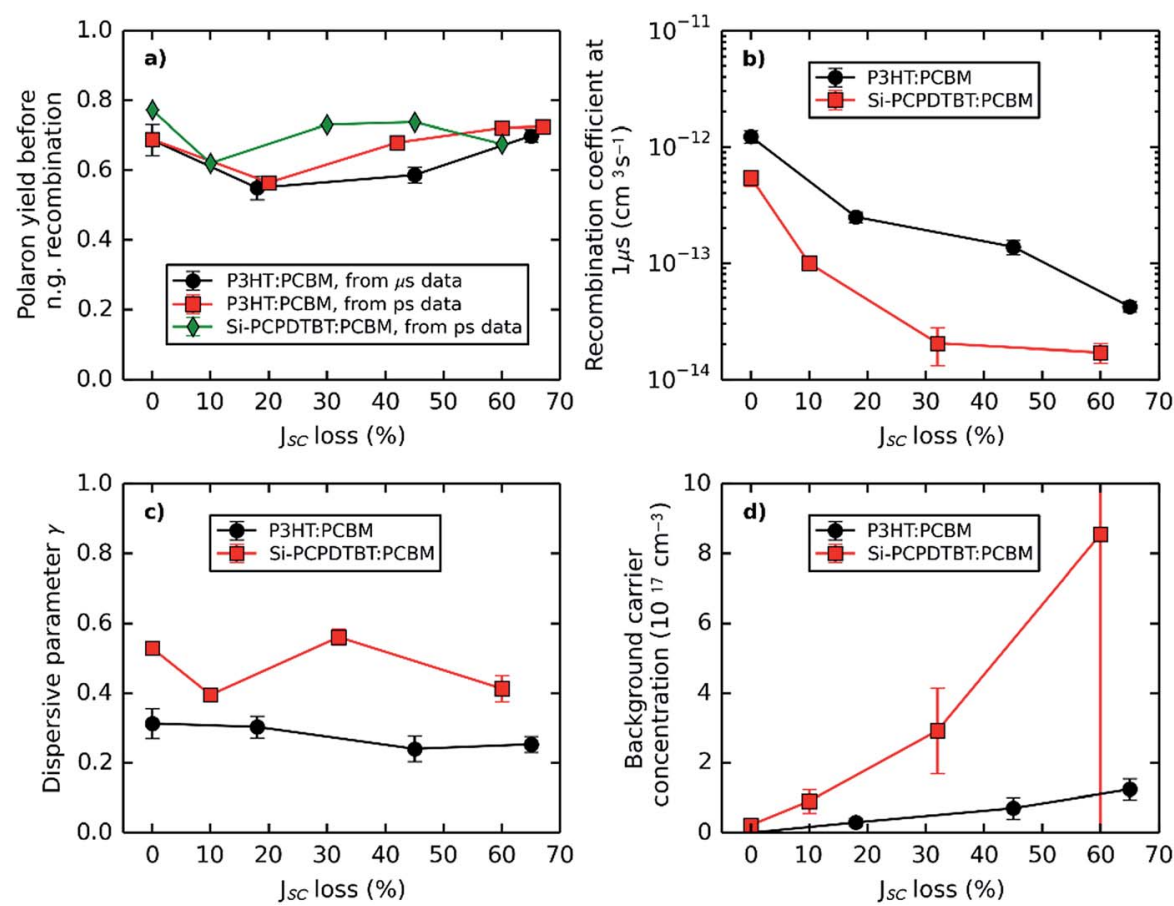

Fig. 4 Fitting parameters from fitting of the curves in Fig. 2 (panel a) and Fig. 3 (panels b-d) using eqn (5). (a) Polaron yield per absorbed photon before non-geminate recombination. Black symbols: absolute data for P3HT:PCBM from microsecond transients (see ESI $\dagger$ ); red symbols: relative data for P3HT:PCBM from femtosecond TA spectra, scaled such that pristine values coincide; blue symbols: absolute data for Si-PCPDTBT from a global analysis of the femtosecond spectra (see Fig. 2). For details see ESI. $\dagger$ (b) Bimolecular recombination coefficient at time $t=1 \mu$ s, assuming a charge absorption cross-section of $10^{-16} \mathrm{~cm}^{2}$ in P3HT and $3 \times 10^{-17} \mathrm{~cm}^{2}$ in Si-PCPDTBT. (c) The exponent of the time-dependent reaction coefficient in eqn (5). (d) Background carrier concentration, assuming the same charge absorption cross-sections as in (b). Panel (d) has been obtained by fitting decay traces after pulsed irradiation (see ESI† for original experiments), all others under the conditions of Fig. 3. 
asymptotic long-time behaviour in the double logarithmic graphs in Fig. 3. For $\gamma>0$, the charge carrier mobility is reduced over time, and the asymptotic slope is between 0 and -1 . The $\gamma$ values displayed in Fig. 4c are around 0.25 and 0.5 for P3HT and Si-PCPDTBT, respectively. They do not show a clear evolution with degradation. Fig. 4d shows the background carrier density, as obtained from the global fits after pulsed irradiation, which is more sensitive to background carriers than CW illumination (for details, see ESI $\dagger$ ). With increasing photo-degradation, a monotonous and strong increase of the background carrier concentration is obtained for both blends. The background carrier concentration in Si-PCPDTBT:PCBM is generally higher than that in P3HT:PCBM, and is nonzero even in the pristine case.

For high background carrier densities and long times, $n \ll c_{\mathrm{b}}$ and hence the square bracket in eqn (4) becomes constant, reducing the effective recombination order to one. The longtime asymptote is therefore no longer a straight line as in the case of a bimolecular reaction, but a stretched exponential, the slope of which bends towards $-\infty$ in a double logarithmic graph. This behaviour can be clearly observed in all panels of Fig. 3, except Fig. 3a, which reports the only experiment with negligible background carrier density.

Fig. 5 presents TA decay curves of photo-induced charges in P3HT:PCBM solar cells of different degrees of degradation under open circuit (OC) and short circuit (SC) conditions, after pulsed irradiation. While under OC conditions, the charge decay is only governed by recombination, under SC conditions both recombination and extraction occur simultaneously. The comparison between OC and SC transients at different degradation levels yields therefore information about the efficiency and the time scale of charge extraction.

In the undegraded sample, the difference between the charge carrier transients under OC and SC conditions is significant, which is in accordance with the short circuit current density of $J_{\mathrm{SC}}=7.5 \mathrm{~mA} \mathrm{~cm}{ }^{-2}$ observed in this device.

When comparing the differently degraded samples, it is readily noticed that the charge carrier concentrations at about 1 $\mu$ s are approximately the same for all samples, irrespective of the extent of degradation, thus confirming that charge carrier formation is not significantly affected by degradation of the active layer. The TA traces measured under OC conditions clearly show that charge carrier recombination slows down considerably upon degradation of the devices and thus confirm the trend observed for the light modulation experiments in films in Fig. 3. While in pristine samples half of the photoinduced charge carriers have recombined after about $60 \mu \mathrm{s}$, it takes several hundred $\mu$ s to reach the same extent of charge carrier loss in the $2 \%$ degraded devices. Also charge carrier extraction becomes slower upon degradation, as obvious from
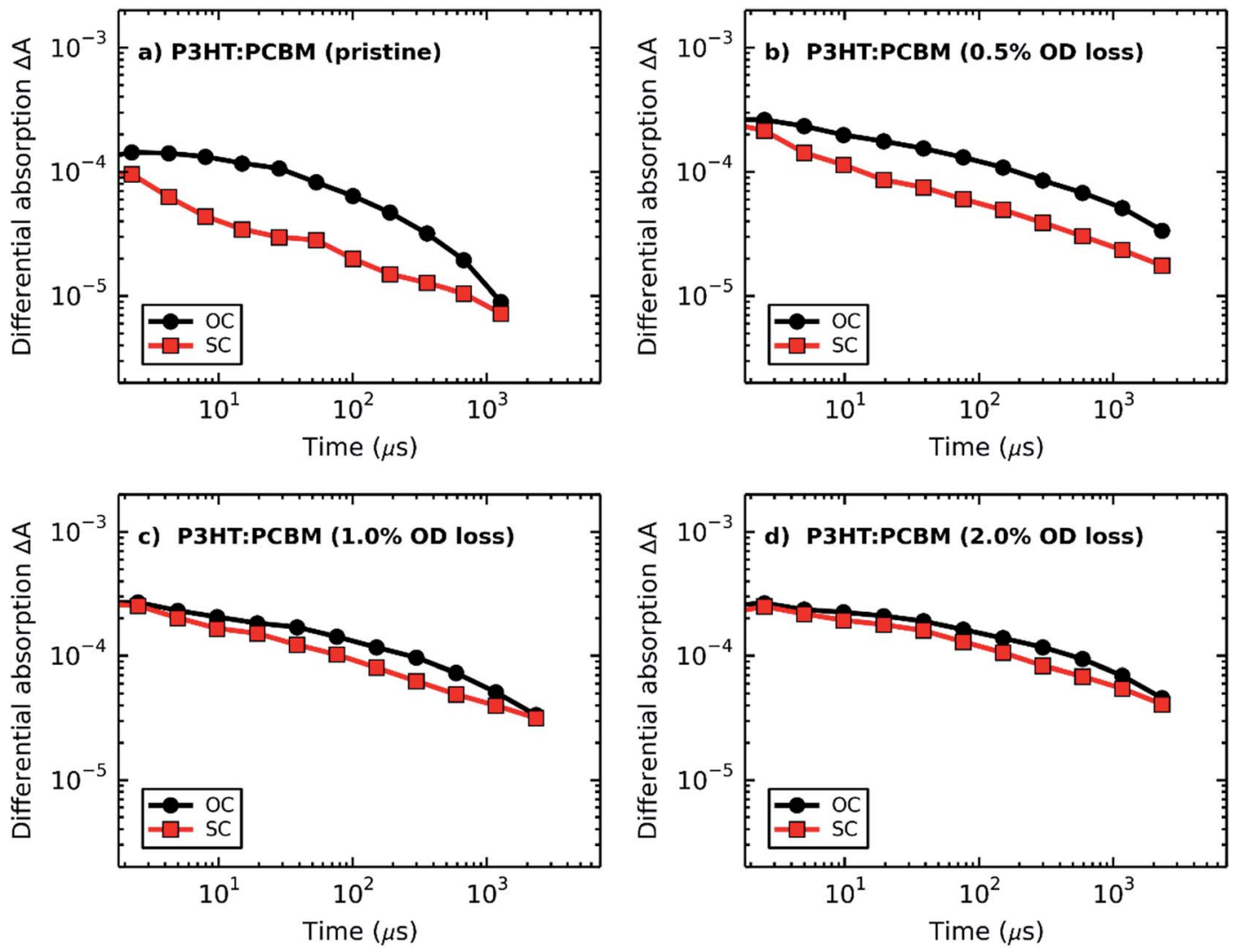

Fig. 5 TA traces in P3HT:PCBM solar cells under open circuit (OC) and short circuit (SC) conditions, at various stages of photo-degradation, as given in the legends. Charge carriers were created by 300 ps pulses at $1.83 \mu \mathrm{J}$ pulse energy in order to achieve initial carrier densities similar to those in Fig. 3a and b; repetition rates were $91 \mathrm{~Hz}$ for panel a and $41 \mathrm{~Hz}$ for all other panels. 
the traces measured under SC conditions. Actually, charge carrier extraction is even more affected than recombination. While $80 \%$ of the charge carriers have disappeared after less than $100 \mu \mathrm{s}$ in the pristine sample, more than $50 \%$ of the charge carriers are still present after this time in the $2 \%$ degraded device. With increasing degradation, the difference between SC and OC measurements, which is significant in pristine cells, becomes very small after $2 \%$ of OD loss (Fig. 5): the maximum ratio of the transient absorption signals under OC and SC conditions is reached after $10 \mu \mathrm{s}, 100 \mu \mathrm{s}$, and $300 \mu \mathrm{s}$ for $0.5,1$, and $2 \%$ OD loss, respectively, thus covering about 2 orders of magnitude.

In summary, photo-oxidation of the active layer hardly affects the charge carrier generation rate, whereas both recombination and extraction are slowed down considerably. The difference between recombination and extraction rates disappears almost completely in heavily degraded devices, which explains the loss of $J_{\mathrm{SC}}$ with progressing degradation. In order to gain some more understanding whether this drastic reduction in extraction efficiency can be explained by the reduction of charge carrier mobility (Fig. 4b) or by the increase of background carrier concentration (Fig. 4d), calculations of the IQE as a function of $\mu, c_{\mathrm{b}}$ and the non-Langevin factor $f_{\mathrm{LV}}$ are presented in Fig. 6 . The curves have been obtained by inserting eqn (3) and (4) into eqn (1) for $\mathrm{d} n / \mathrm{d} t=0$ (steady state) and then relating the rate of extraction to the rate of generation. Since eqn (3) refers to Langevin (i.e., exclusively drift driven) recombination in homogeneous three-dimensional space, while, e.g., carrier transport in $\mathrm{P} 3 \mathrm{HT}$ :PCBM has been proposed to be quasi$2 \mathrm{D},{ }^{28}$ the results in Fig. 6 are to be considered as instructive approximations. It is also important to highlight that the effective charge mobility $\mu_{\text {eff }}$ in eqn (1b) is microscopic, while typically charge carrier mobilities are measured

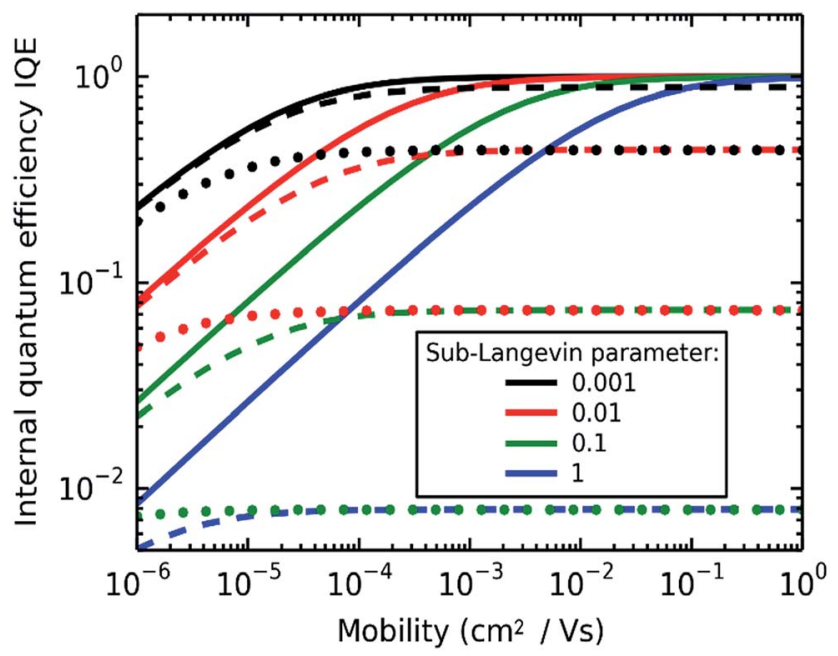

Fig. 6 Simulation of internal quantum efficiency (IQE) as a function of effective charge carrier mobility for various values of the sub-Langevin parameter $f_{\mathrm{LV}}$, according to eqn (1a) and (4). The background carrier concentration $c_{b}$ was set to $0,10^{17}$ and $10^{18} \mathrm{~cm}^{-3}$ (solid, dashed and dotted curves, respectively). The fixed parameters were: 1 absorbed sun, built-in voltage $0.6 \mathrm{~V}$, electrode spacing $d=250 \mathrm{~nm}$. macroscopically using techniques like CELIV. ${ }^{29}$ In BHJ materials, the presence of grain boundaries and limited percolation paths might reduce the macroscopic mobility against the microscopic one. Consequently, extraction might be penalized against recombination. Therefore the curves in Fig. 6 should be understood as upper limits for IQE at the given parameters.

Solid curves refer to zero background carrier density and thus reflect the dependence of IQE on $\mu$ for a reaction order of two. Assuming a reasonable built-in field, it is found that the IQE for $c_{\mathrm{b}}=0$ drastically depends on the non-Langevin factor: for $f_{\mathrm{LV}}=1$, a mobility reduction from $10^{-3}$ to $10^{-4} \mathrm{~cm}^{2} \mathrm{~V}^{-1} \mathrm{~s}^{-1}$ suffices to bring down the IQE from $23 \%$ to only $8 \%$. On the other hand, if $f_{\mathrm{LV}}$ is 0.001 (recombination rate is $1 / 1000$ of the one expected from Langevin kinetics), then IQE remains at 56\% even for mobilities as low as $10^{-5} \mathrm{~cm}^{2} \mathrm{~V}^{-1} \mathrm{~s}^{-1}$. We conclude that a mobility reduction of more than an order of magnitude, as conjectured from Fig. 4b, significantly reduces IQE, as long as non-Langevin factors are close to unity. The presence of background carriers further reduces IQE, compare solid, dashed, and dotted curves of the same colour, respectively. For P3HT with its low non-Langevin factor, background carrier concentrations up to $10^{17} \mathrm{~cm}^{-3}$ (as obtained in Fig. 4c) do not cause a significant lowering of IQE. The situation is different for SiPCPDTBT, where background carrier densities close to $10^{18}$ $\mathrm{cm}^{-3}$ have been found. Since also the non-Langevin factor is lower in this material $f_{\mathrm{LV}}=0.5$ has been published as a lower limit), background carriers can have a strong effect on IQE in this material. ${ }^{30}$ In summary, Fig. 6 shows that in the case of SiPCPDTBT reduced mobility and enhanced background carrier concentration easily account for the observed loss of $J_{\mathrm{SC}}$ upon photo-oxidation of the active layer (see Fig. 1). The situation is different for P3HT:PCBM. Because of the low non-Langevin factor in P3HT, even the combination of both effects shown in Fig. 6 does not fully explain the strong $J_{\mathrm{SC}}$ reduction observed in Fig. $1 .^{29}$ Therefore, it might be necessary to invoke additional degradation effects to account for the $J_{\mathrm{SC}}$ loss, e.g., the reduction of the built-in field. Such a reduction of the built-in field is indeed expected from the presence of mobile background carriers, as they form a space charge region shielding the builtin potential, similar to the case of reversible performance degradation in P3HT:PCBM. ${ }^{13}$

\section{Summary}

We have analyzed charge transients in films of P3HT:PCBM and Si-PCPDTBT:PCBM on a femtosecond to millisecond time scale as a function of photo-degradation in order to understand the reason for the electrical performance loss due to irreversible photo-oxidation which is one of the limiting factors of device lifetime in inverted OSC structures. For both polymer:PCBM blends, the charge generation rate hardly changes during photo-oxidation, suggesting that the charge separation coefficient is practically independent of the level of degradation. However, in P3HT:PCBM, oxygen induced degradation causes a strong reduction of the charge recombination coefficient, which formally corresponds to a reduction of charge carrier mobility. This is in stark contrast to inorganic semiconductors where 
often a defect-enhanced recombination rate is found. ${ }^{23}$ Concomitantly, the order of charge carrier recombination is reduced from values of close to two in pristine P3HT:PCBM films to values close to unity already at degradation levels as low as $0.5 \%$ OD loss. The extraction rate of charge carriers is even more seriously reduced by photo-degradation than expected from the mobility changes calculated from the recombination term, which leads to the loss of short circuit current in degraded devices. While the rate of extraction exceeds that of recombination by two orders of magnitude in pristine samples, both rates are more or less equal in strongly degraded samples. The calculation of the IQE reduction as a function of $f_{\mathrm{LV}}, c_{\mathrm{b}}$, and $\mu$ shows that at reasonable values of $f_{\mathrm{LV}}, \mu$ plays a stronger role than $c_{\mathrm{b}}$, but both effects might not be sufficient to explain the strong $J_{\text {SC }}$ reduction observed upon photo-oxidation. We suggest that the increased background carrier density introduced by irreversible degradation contributes to the loss of short circuit current by shielding the extraction field in the bulk of the devices, which corresponds to a decrease of $E_{\mathrm{bi}}$ in eqn (1) and thus leads to an additional decrease of the extraction term. This additional decrease leads to a longer dwelling time of the photo-generated carriers in the device so that in turn their recombination is significantly enhanced in the pseudo-first order reaction observed in our TA measurements, similar to the effect that has been shown to be operative in reversibly degraded P3HT:PCBM solar cells. ${ }^{13}$

\section{Acknowledgements}

The research leading to these results has received funding from the European Union Seventh Framework Programme (FP7/2011 under grant agreement ESTABLIS no. 290022) and was partially funded by the BMBF project "POPUP" (FKZ 03EK3501C). LL thanks the Spanish Economic Ministry (MINECO) for a Ramon y Cajal fellowship and the EU for financial support via the COFUND program AMAROUT, and the Community of Madrid (Projects MADRISOLAR-2 and NANOBIOMAGNET). RW thanks the Community of Madrid (Project NANOBIOMAGNET). H.-J. E. acknowledges financial support by the Solar Fab of the Future on the Energy Campus Nürnberg (Bavarian State Government Grant no. 20-3043.5).

\section{Notes and references}

1 F. C. Krebs, S. A. Gevorgyan and J. Alstrup, J. Mater. Chem., 2009, 19, 5442.

2 M. Jorgensen, K. Norrman, S. Gevorgyan, T. Tromholt, B. Andreasen and F. Krebs, Adv. Mater., 2012, 24, 580.

3 M. Jørgensen, K. Norrman and F. C. Krebs, Sol. Energy Mater. Sol. Cells, 2008, 92, 686.

4 N. Grossiord, J. Kroon, R. Andriessen and P. Blom, Org. Electron., 2012, 13, 432.

5 J. Lee, J. Jung, J. Jo and W. Jo, J. Mater. Chem., 2012, 22, 24265.

6 C. J. Brabec, Sol. Energy Mater. Sol. Cells, 2004, 83, 273.

7 J. M. J. Frèchet and B. C. Thompson, Angew. Chem., Int. Ed., 2008, 47, 58 .
8 B. van der Wiel, H.-J. Egelhaaf, H. Issa, M. Roos and N. Henze, MRS Proc., 2014, 1639, DOI: 10.1557/opl.2014.88.

9 J. A. Hauch, P. Schilinsky, S. A. Choulis, S. Rajoelson and C. J. Brabec, Appl. Phys. Lett., 2008, 93, 103306.

10 J. A. Hauch, P. Schilinsky, S. A. Choulis, R. Childers, M. Biele and C. J. Brabec, Sol. Energy Mater. Sol. Cells, 2008, 92, 727.

11 J. Schafferhans, A. Baumann, A. Wagenpfahl, C. Deibel and V. Dyakonov, Org. Electron., 2010, 11, 1693.

12 H. Hintz, H.-J. Egelhaaf, L. Lüer, J. Hauch, H. Peisert and T. Chassé, Chem. Mater., 2011, 23, 145.

13 A. Seemann, T. Sauermann, C. Lungenschmied, O. Armbruster, S. Bauer, H.-J. Egelhaaf and J. Hauch, Sol. Energy, 2010, 85, 1238.

14 F. Deschler, A. De Sio, E. von Hauff, P. Kutka, T. Sauermann, H.-J. Egelhaaf, J. Hauch and E. Da Como, Adv. Funct. Mater., 2012, 22, 1461.

15 J. Guo, H. Ohkita, H. Benten and S. Ito, J. Am. Chem. Soc., 2010, 132, 6154.

16 M. Koppe, H.-J. Egelhaaf, E. Clodic, M. Morana, L. Lüer, A. Troeger, V. Sgobba, D. M. Guldi, T. Ameri and C. J. Brabec, Adv. Energy Mater., 2013, 3, 949.

17 G. Juška, K. Genevičius, N. Nekrašas, G. Sliaužys and R. Österbacka, Appl. Phys. Lett., 2009, 95, 013303.

18 I. A. Howard, R. Mauer, M. Meister and F. Laquai, J. Am. Chem. Soc., 2010, 132, 14866.

19 C. Deibel, D. Rauh and A. Foertig, Appl. Phys. Lett., 2013, 103, 043307.

20 R. Pacios, A. J. Chatten, K. Kawano, J. R. Durrant, D. D. C. Bradley and J. Nelson, Adv. Funct. Mater., 2006, 16, 2117.

21 S. Neugebauer, J. Rauh, C. Deibel and V. Dyakonov, Appl. Phys. Lett., 2012, 100, 263304.

22 (a) C. G. Shuttle, B. O'Regan, A. M. Ballantyne, J. Nelson, D. D. C. Bradley, J. de Mello and J. R. Durrant, Appl. Phys. Lett., 2008, 92, 093311; (b) C. G. Shuttle, B. O'Regan, A. M. Ballantyne, J. Nelson, D. D. C. Bradley and J. R. Durrant, Phys. Rev. B: Condens. Matter Mater. Phys., 2008, 78, 1132012.

23 W. Shockley and W. T. Read Jr, Phys. Rev., 1952, 87, 835.

24 B. Movaghar, M. Grünewald, B. Ries and H. Bässler, Phys. Rev. B: Condens. Matter Mater. Phys., 1986, 33, 5545.

25 C. Silva, A. S. Dhoot, D. M. Russell, M. A. Stevens, A. C. Arias, J. D. MacKenzie, N. C. Greenham and R. H. Friend, Phys. Rev. B: Condens. Matter Mater. Phys., 2001, 64, 125211.

26 J. Nelson, Phys. Rev. B: Condens. Matter Mater. Phys., 2003, 67, 155209.

27 P. Langevin, Ann. Chim. Phys., 1903, 28, 433.

28 A. V. Nenashev, F. Jansson, S. D. Baranovskii, R. Österbacka, A. V. Dvurechenskii and F. Gebhard, Appl. Phys. Lett., 2010, 96, 213304.

29 A. Pivrikas, N. S. Sariciftci, G. Juška and R. Österbacka, Prog. Photovoltaics, 2007, 15, 677.

30 T. M. Clarke, D. B. Rodovsky, A. A. Herzing, J. Peet, G. Dennler, D. DeLongchamp, C. Lungenschmied and A. J. Mozer, Adv. Energy Mater., 2011, 1, 1062. 